\title{
Relativity of Speed of Light with Speed of Universe Expansion
}

\author{
Dhiren Sampat \\ Mumbai University, Mumbai, India \\ Email: dmsampat@yahoo.com
}

Received 25 May 2014; revised 21 June 2014; accepted 11 July 2014

Copyright (C) 2014 by author and Scientific Research Publishing Inc.

This work is licensed under the Creative Commons Attribution International License (CC BY). http://creativecommons.org/licenses/by/4.0/

c) (i) Open Access

\begin{abstract}
Theory of "Relativity of speed of light with speed of universe expansion" explains relation of speed of light with speed of universe expansion. This theory provides an evidence that the time cannot be relative as stated by Theory of General Relativity. Theory of Special Relativity and Theory of General Relativity were based on two fundamental propositions i.e. constancy of speed of light and independence of physical laws (especially the constancy of speed of light) from the choice of inertial system. However, postulate of these theories is not correct. Theory of "Relativity of speed of light with speed of universe expansion" answers fundamental propositions i.e. constancy of speed of light and independence of physical laws in logical manner. This theory also explains real reason behind $E=m c^{2}$.
\end{abstract}

\section{Keywords}

Speed of Light, Theory of General Relativity, Theory of Special Relativity, $E=m c^{2}$

\section{Introduction}

There are three basic questions related to speed of light.

1) From where light photon which does not have even intrinsic mass gets energy to move at speed of approximately $300,000 \mathrm{~km} / \mathrm{s}$;

2) How come speed of gravitational force which is based on mass of an object is same as speed of light; and

3) Why when mass is released as energy, the energy released is related to speed of light (c) ( $\left.E=m c^{2}\right)$.

What is the significance of speed of light in these cases? Or is it that all these are related to one common source (universe expansion) and hence derive speed/energy from the same.

Let us study this in detail. We will also get explanations regarding

1) Why light moves in all directions at same speed; 
2) Reason why speed of light does not depend on speed of source of light;

3) Reason behind Time Dilatation at higher speed; and

4) Real reason behind $E=m c^{2}$

We will also validate our observations with Hubble’s constant.

\section{Universe Expansion}

\subsection{Understanding Expansion of Universe and Location of Our ("Milky Way") Galaxy in Universe}

After big bang universe started expanding in all directions [1]. We can think of this expansion as a sphere (like rubber ball) which is being stretched in all directions and thus expanding. The galaxies including galaxy in which we reside move away from the "center of universe" (i.e., position of big bang) on account of universe expansion. The speed at which galaxy would be moving away from center of universe, is related to speed at which universe is expanding and position of galaxy with respect to "center of universe" and "outmost layer of universe".

This can be explained with the help of following diagrams (Diagram 1):

Diagram 1 represents position at the time of big bang.

Diagram 2 represents universe after lapse of time $t$ where universe is expanding at the rate of $2 x$ per time of $t$. (at this point to keep things simple we will not assign value to $\mathrm{x}$ ). Our galaxy which is lying at midpoint of "center of universe" and "outmost layer of universe" would have moved $\mathrm{x}$ on account of expansion of universe by $2 \mathrm{x}$.

Now let us have diagram showing position at the time of $2 \mathrm{t}$.

As shown in Diagram 3, universe would have been expanded to 4x and position of our galaxy would be at 2x. Thus universe is expanded ("stretched") at the rate of $x$ per $t$ on both sides of our galaxy i.e. side which is towards "center of universe" as well as side which is towards "outmost layer of universe". When we apply this to sphere (like rubber ball) it can be seen that universe would have expanded at the rate of $\mathrm{x}$ in all directions with respect to galaxy as shown in Diagram 4.

Now on account of this expansion (stretching), light photon which does not have any intrinsic mass moves along with universe expansion in all directions. Other objects including us, which have basic mass remain attached to galaxy on account of gravitational force and hence do not move along with universe expansion in the manner light moves.

Light is made up of large number of photons. A I-watt night light emits a billion each seconds. These photons move alongwith universe expansion in all directions. This explains dual character of light photons which are particle and move as wave alongwith universe expansion in all directions.

Now $\mathrm{x}$ is the speed at which universe is expanding in all directions with respect to galaxy, which we measure as speed of light, which is approximately $300,000 \mathrm{~km} / \mathrm{s}$. Now as can be seen from above diagrams, our galaxy is also moving at the speed of approximately $300,000 \mathrm{~km} / \mathrm{s}$. Gravitational force of our galaxy also moves with our

Diagram 1. Universe at the time of big bang.

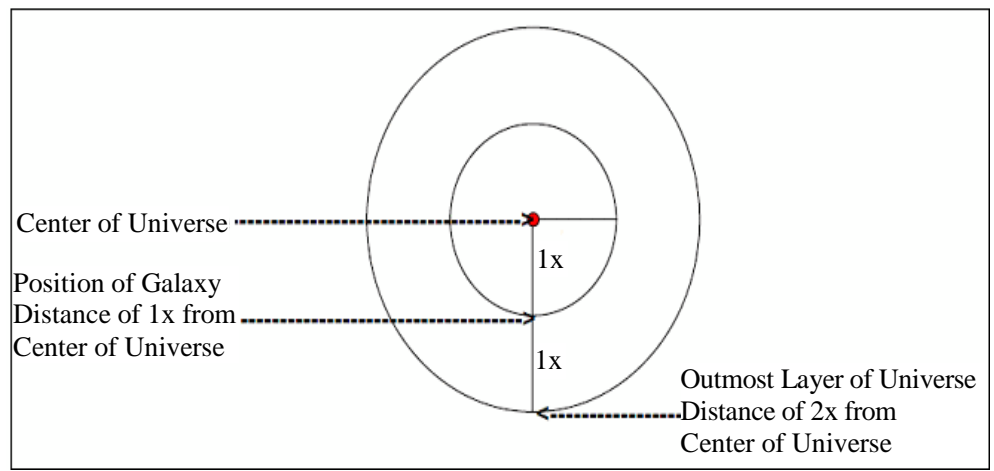

Diagram 2. Universe after lapse of time t. 


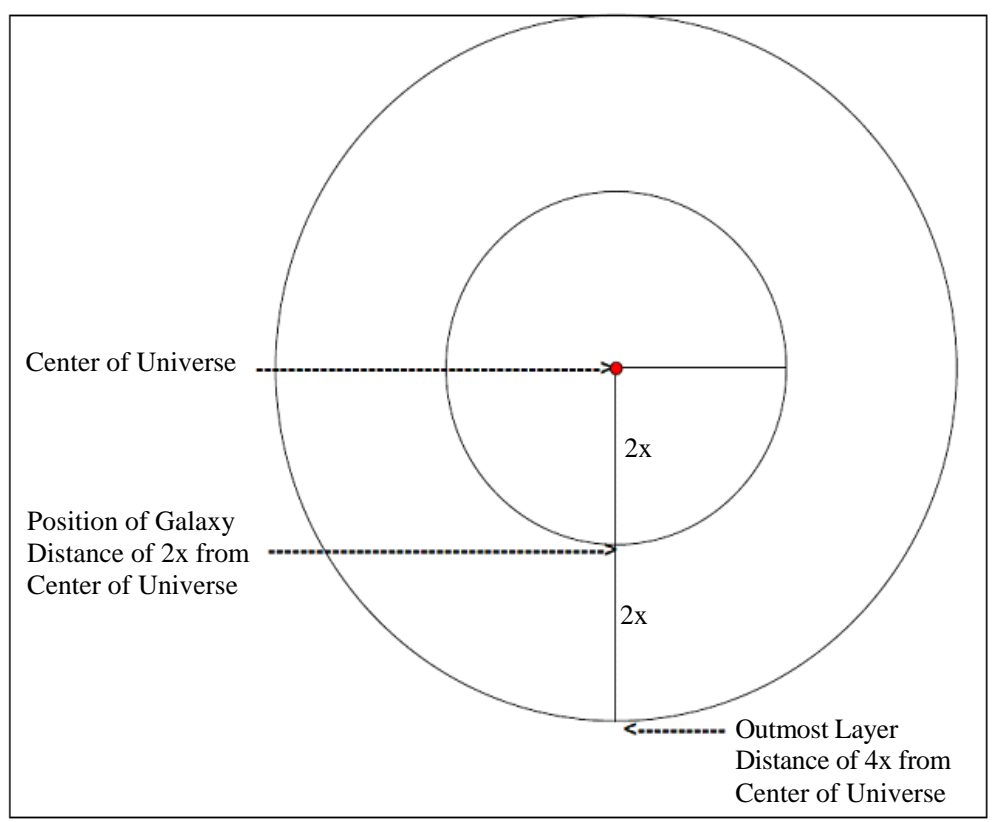

Diagram 3. Universe after lapse of time 2t.

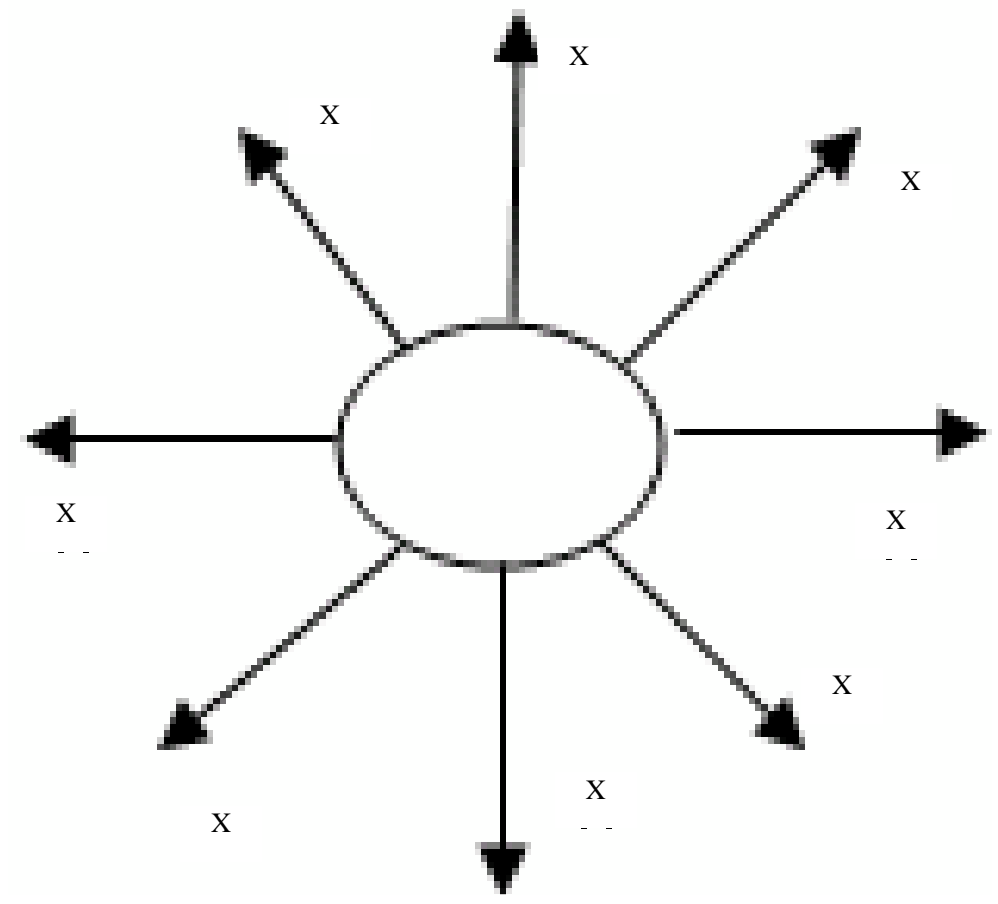

Diagram 4. Expansion of universe with respect to galaxy.

galaxy at the same speed as speed of our galaxy. Thus we observe speed of light and speed of gravitational force as same.

\subsection{Implication of Universe Expansion on Speed of Gravitational Force}

As stated by general theory of relativity the speed of gravitational waves is equal to the speed of light in vacuum [2]. Gravity is curvature in space. Speed of this curvature should be related to the speed of object ("galaxy") which is causing this curvature. Imagine a heavy ball rolling on rubber mattress. Curvature caused by this ball 
on rubber matter is similar to gravity. Now speed of the curvature caused by this ball is exactly equal to speed of ball. Similarly speed of gravitational waves is equal to speed of our galaxy.

As stated above, Milky Way galaxy moves at speed of approximately 300,000 km/s on account of universe expansion. Curvature caused by Milk Way also moves at the same speed alongwith Milky Way Galaxy.

In later part we will see how c in equation $E=m c^{2}$, refers to the speed of object ("galaxy"), and energy released is in line with kinetic energy formula where energy is related to velocity of object and mass of object, $E=$ $M v^{2}$.

\section{Evidences Supporting Shape of Universe Expansion and Location of Milky Way Galaxy}

In the above section we made following propositions regarding shape of Universe Expansion, location of Milky Way Galaxy and speed of Milky Way Galaxy.

- Universe is expanding in shape of Sphere;

- Milky Way Galaxy is located at the mid-point of center of Universe and outmost layer of Universe;

- Our Galaxy is moving at speed of approximately $300,000 \mathrm{~km} / \mathrm{s}$ on account of universe expansion.

There are two strong evidences to suggest that universe is expanding in sphere and our galaxy is at mid point of center of universe and outmost layer of universe

\subsection{Hubble's Constant}

Value of Hubble constant reinforce our understanding that universe is expanding in sphere (like shape of rubber ball) and our galaxy is at midpoint of "center of universe" and "outmost layer of universe" otherwise value of Hubble's constant cannot be constant (i.e. uniform). This is only possible in structure like sphere where our galaxy is at midpoint of "center of sphere" and "outmost layer of sphere". Refer diagram 2 and 3 shown above, speed at which any point (galaxy) is moving away from our galaxy is directly proportional to the distance of that point (galaxy) to our galaxy.

\subsection{Cosmic Microwave Background Radiation (CMBR)}

Cosmic background radiation is well explained as radiation left over from an early stage in the development of the universe, and its discovery is considered a landmark test of the Big Bang model of the universe. With a traditional optical telescope, the space between stars and galaxies (the background) is completely dark. However, a sufficiently sensitive radio telescope shows a faint background glow, almost exactly the same in all directions, that is not associated with any star, galaxy, or other object [3]. The CMB temperature on the sky is remarkably uniform. At the level of 1 part in 1000, the CMB temperature varies.

Both these strongly support that universe is expanding in sphere (like rubber ball) and our galaxy is at center of sphere (universe) and outmost layer of universe. If universe was not expanding like sphere and our galaxy would not have been at mid-point of center of sphere (universe) and outmost layer of universe, these would not have been possible.

\section{Evidences Supporting Speed of Our Galaxy}

\subsection{Measuring Speed of Our Galaxy through Hubble's Constant}

In the years following proof of existence of other galaxies, Hubble spent his time cataloging their distances and observing their spectra. At that time most people expected the galaxies to be moving around randomly, and so expected to find as many blue-shifted spectra as red-shifted ones. It was quite a surprise, therefore, to find that most of galaxies appeared red-shifted: nearly all were moving away from us! More surprising still was the finding that Hubble published in 1929: even the size of a galaxy's red shift is not random, but it is directly proportional to the galaxy's distance from us. Or, in other words, the farther a galaxy is, the faster is moving away [4]!

At present Hubble's constant value is estimated to be about $70 \mathrm{kms}^{-1} \cdot \mathrm{Mpc}^{-1}$ [5]. It is stated that distance of our galaxy from center of universe is equal to $1.40 \times 10^{10}$ light years or about fourteen thousand million light years. Therefore velocity of recession (i.e. speed of our galaxy) from center of universe is given by

$$
\text { Velocity of recession }(v)=\text { Hubble constant }(H) \times \text { distance }(r)
$$




$$
V=\frac{70 \mathrm{~km} / \mathrm{s}}{1 \mathrm{Mpc}} \times 1.4 \times 10^{10} \text { light years }
$$

$1 \mathrm{Mpc}=3,260,000$ light-years [6]

Replacing value of $1 \mathrm{Mpc}$ in above equation we get

$$
\begin{aligned}
& V=\frac{70 \mathrm{~km} / \mathrm{s}}{3,260,000 \text { lightyears }} \times 1.4 \times 10^{10} \text { light years } \\
& V=70 \mathrm{~km} / \mathrm{s} \times 4294.48 \\
& V=300,613.6 \mathrm{~km} / \mathrm{s}
\end{aligned}
$$

Therefore speed of our Galaxy from center of universe is approximately 300,613.6 km/s, this is approximately the same speed as we stated above.

\subsection{Speed of Gravitational Force}

As stated by "Theory of General Relativity" the speed of gravitational waves is equal to the speed of light in vacuum. Even experiments have confirmed speed of gravity approximately equal to speed of light. Gravity is curvature in space. Speed of this curvature should be related to the speed of object ("galaxy") which is causing this curvature. Imagine a heavy ball rolling on rubber mattress. Curvature caused by this ball on rubber mattress is similar to gravity. Speed of the curvature caused by this ball is exactly equal to speed of ball. If ball moves at the speed of $1 \mathrm{~km} / \mathrm{s}$ then curvature also will move alongwith ball at the same speed. Similarly speed of gravitational waves is equal to speed of our galaxy.

As stated above, Milky Way galaxy moves at speed of approximately 300,000 km/s on account of universe expansion. Curvature caused by Milk Way also moves at the same speed alongwith Milky Way Galaxy.

To analyze this further let us have a case where a particular galaxy is moving at speed of 100,000 km/s then speed of curvature ("gravity") caused by that Galaxy would be 100,000 km/s. If the speed of gravity of that galaxy is not $100,000 \mathrm{~km} / \mathrm{s}$ but is equal to the speed of light i.e. $299,792 \mathrm{~km} / \mathrm{s}$ then it may lead to situation where galaxy is at one location in universe while curvature caused by that galaxy (gravity) is at different location in Universe which cannot be possible. Speed of Gravitational force of our Galaxy which is equal to the speed of light gives us strong evidence that our galaxy is moving at the speed of approximately $299,792 \mathrm{~km} / \mathrm{s}$ in universe.

\subsection{Energy Generated by Stars of Milky Way Galaxy}

As explained above, velocity of MILKY WAY galaxy on account of universe expansion is approximately $299,792 \mathrm{~km} / \mathrm{s}(\mathrm{v})$. Kinetic Energy is related to mass $(M)$ and velocity of mass $(v)$. Mass requires energy to attain velocity. Therefore when mass is released as energy, energy generated is related to mass $(M)$ and velocity of mass $(v)$. This is in line with law of conservation of energy which states that the total amount of energy in an isolated system remains constant over time. If energy generated is not related to velocity of mass $(v)$ but to speed of light $(c)$, then the above law cannot be complied with. Therefore energy generated by Stars of MILKY WAY galaxy is on account of velocity of MILKY WAY galaxy on account of universe expansion i.e. $E=M v^{2}$.

If we analyse carefully the way $E=m c^{2}$ is derived we can make out that $E$ refers to Energy of light photon and $\mathrm{m}$ refers to mass of light and not the mass of the object and $\mathrm{c}$ as we are aware refers to speed of light in $E=$ $m c^{2}$. Therefore,

$$
\left.E(\text { Energy of light photon })=m \text { (mass of light }) \times c^{2} \text { (where } c \text { is speed of light }\right)
$$

Now replacing light with an object in above equation we have

$$
E(\text { Energy of object })=M(\text { mass of object }) \times v^{2}(\text { where } v \text { is speed of object })
$$

Thus,

$$
\begin{aligned}
& E=m c^{2} \text { is nothing but equation of kinetic energy which is } \\
& E \text { (Energy of object) }=M \text { (mass of object) } \times v^{2} \text { (where } v \text { is velocity of object) }
\end{aligned}
$$


This has been explained in detail in Section 6 .

\section{Review of Existing Theories (Theory of Special Relativity and Theory of General Relativity) and Evidences Suggesting Why These Theories Do Not Reflect Correct Picture}

\subsection{Why Time Can Not Be Relative in Universe}

As a consequence of universe expansion all galaxies are moving away from center of universe. Now say if particular galaxy is moving at speed of $99.99 \%$ of speed of light. If time is related to speed of object in that case time for object (galaxy) moving at such high speed should slow down. One of the consequences of time, on account of universe expansion is, galaxy should move away from the "center of universe" with elapse of time, but if time is relative, time should slow down at such high speed and hence galaxy should move away from "center of universe" at slow speed. Both these i.e. time getting slower on account of high speed of galaxy and simultaneously galaxy moving at slow speed on account of time running relatively slower are contradictory.

Now let us understand why even within our galaxy, in our solar system time cannot be relative.

\subsection{Time Which Is Measured Based Independent Source Will Remain Universal for All Observers Irrespective of Speed of Observers}

To understand this let us have a case of three observers, A, B and C. A-who is staying in New York city and not moving i.e. speed of zero. B-who is traveling in a train which is moving around New York city at $10 \%$ of speed of light and $\mathrm{C}$-who is traveling in a space rocket with a speed of $10 \%$ of speed of light i.e. same speed as speed of B. Now, Day/year for A and B, who both are in New York city will be same, Earth's orbital speed will not change for B even if B is traveling at $10 \%$ of speed of light. Earth will complete one Day/Year in same time for A and B. Thus planetary time will run universally for A and B.

Now let us analyze what will happen to C. If time were to depend on speed of observer than time will have to be same for B and C as both B and C are traveling at the same speed that is $10 \%$ of speed of light. As explained above, planetary time for B is same as planetary time for A, which means planetary time for A and C is also same. Thus time which is measured based on independent source is universal for all observers irrespective of speed of observers.

\subsection{Let Us Understand Why Clock Slows down at Higher Speed}

With increase in speed of clock, gravitational mass of clock increases, resulting in relatively slower movements of clock. However, it may be noted that the slower movement would be applicable for that clock only. This will not have any impact on time which is measured based on independent source. The phenomenon is same as with increase in weight more energy is required to achieve same speed. However, if energy is kept constant than speed slows down. This can be explained with the help of following example. Say if two motors-motor A and motor B are running at speed of $50 \mathrm{~km}$ per hour. If weight of motor $\mathrm{A}$ is increased while keeping other factors constant, speed of motor A slows down compared to speed of motor B. Similarly, in case of two clocks, clock A and clock B, when mass of clock A is increased, speed of clock A slows down. This is also applicable for atomic clocks where due to increase in mass speed slows down. However, as explained above, this will not have any impact on time which is measured based on independent source.

\subsection{Gravitational Time Dilation}

Following argument is given in support of theory of General Relativity. There are identical atomic clocks at the Royal Observatory in Greenwich England and the National Bureau of standards in Boulder Colorado. The one in Greenwich England ticks 5 microseconds/year slower than the one in Boulder Colorado which is predicted by General Relativity given their one mile difference in altitude. The clocks are accurate to 1 microsecond/year so the difference cannot be a result of error [7].

However, since millions of years day/year remains same at both locations. If this is the case, can we say that time runs differently at both locations? Or time runs slower near high gravitational field. The answer as explained above is when gravitational mass increases speed slows down. However, it may be noted that the slower 
movement would be applicable for that clock only. This will not have any impact on time which is measured based on independent source.

\subsection{Characteristics of Light}

Let us explain characteristic why "Speed of light does not depend on speed of source of light". As explained above, presently universe is expanding at the rate of $2 \mathrm{x}(600,000 \mathrm{~km})$ per t (second). Milky Way galaxy on account of universe expansion moves at the rate of $300,000 \mathrm{~km} / \mathrm{s}$. Further, universe around our galaxy expands at the rate of $300,000 \mathrm{~km} / \mathrm{s}$ in all directions as explained above. As a result, light moves in all directions at the speed of 300,000 km/s. Speed of universe expansion with respect to galaxy would be same irrespective of speed of source of light therefore speed of light does not depend on speed of source of light.

\section{Understanding $E=m c^{2}$ and Evaluating Whether Energy Generated Is on Account of $E=M v^{2}$ or on Account of $E=m c^{2}$}

\subsection{Implication of Universe Expansion on $E=m c^{2}$}

Our galaxy is moving on account of universe expansion at the rate of $300,000 \mathrm{~km} / \mathrm{s}$. Kinetic Energy is related to mass $(M)$ and velocity of mass $(v)$. Mass requires energy to attain speed. Therefore when mass is released as energy, energy generated is related to mass $(M)$ and velocity of mass $(v)$. This is in line with law of conservation of energy which states that the total amount of energy in an isolated system remains constant over time. If energy generated is not related to velocity of mass $(v)$ but to speed of light $(c)$, then the above law cannot be complied with. Thus above equation needs to be changed to $E=M v^{2}$ where $v$ is velocity of mass. It may be noted that result would be same since as explained above masses (our galaxy) on account of universe expansion are moving at speed of approximately $300,000 \mathrm{~km} / \mathrm{s}$.

\subsection{Derivation of $E=m c^{2}$}

If we read carefully the way $E=m c^{2}$ is derived we can make out $\mathrm{m}$ in $E=m c^{2}$ refers to mass of light and not mass of object. Therefore,

$$
\left.E(\text { Energy })=m \text { (mass of light }) \times c^{2} \text { (where } c \text { is speed of light }\right)
$$

Now replacing light with an object in above equation we have

$$
E \text { (Energy) }=m \text { (mass of object }) \times v^{2} \text { (where } v \text { is speed of object) }
$$

Thus, $E=m c^{2}$ is nothing but equation of kinetic energy which is

$$
E=M \text { (mass of object) } \times v^{2} \text { (where } v \text { is velocity of object) }
$$

A question may arise that formula for kinetic energy is $1 / 2 M v^{2}$ and not $M v^{2}$. Let us understand why in this case $M v^{2}$ and not $1 / 2 M v^{2}$. Kinetic energy formula of $1 / 2 M v^{2}$ is arrived based on average velocity that is velocity starting with zero and velocity reaching its peak and thus average velocity is $1 / 2$. However in this case, velocity of our galaxy continues to be same i.e. approximately $300,000 \mathrm{~km} / \mathrm{s}$ and hence average velocity is also $\mathrm{v}$ and not $1 / 2 v$. Hence in this case kinetic energy equation would be $M v^{2}$.

Let us derive kinetic energy formula in this case

We know that Force $=$ mass $\times$ acceleration

$$
F=M \times a
$$

where $F$ represents net force

$$
M=\text { Mass of object }
$$

Acceleration is velocity/time

Therefore,

$$
a=\text { acceleration }=v / t
$$

where, 


$$
v=\text { Velocity of mass }
$$

and

$$
t=\text { time taken }
$$

Distance is velocity $\times$ time

Therefore,

$$
d=v \times t
$$

where,

$$
\begin{aligned}
& d=\text { Distance } \\
& v=\text { Velocity }
\end{aligned}
$$

And

$$
t=\text { time taken }
$$

Now we know that

$$
\text { Kinetic Energy }=F \times d
$$

Kinetic Energy $=M \times a \times d($ since $F=M a)$.

Therefore,

$$
\begin{gathered}
\text { Kinetic Energy }=M \times v / t \times v t \\
\text { Kinetic Energy }=M \times v^{2} .
\end{gathered}
$$

\section{References}

[1] Hawking, S. (1988) A Brief History of Time, the Expanding Universe. Bantam Press, UK, 42.

[2] Speed of Gravity, The Speed of Gravitational Waves in the General Theory of Relativity Is Equal to the Speed of Light in Vacuum. http://en.wikipedia.org/wiki/Speed_of_gravity

[3] Cosmic Microwave Background Radiation. http://en.wikipedia.org/wiki/Cosmic microwave background radiation

[4] Hawking, S. (1988) A Brief History of Time from Big Bang to Black Holes-The Expanding Universe. Bantam Press, UK, 41.

[5] Lifting the Veil on Hubble's Constant Ten +1 Measurements of the Hubble Constant. http://science.nasa.gov/science-news/science-at-nasa/1999/ast25may99_2/

[6] Parsec Megaparsecs and Gigaparsecs. http://en.wikipedia.org/wiki/Parsec

[7] Gravitational Time Dilation. http://genesismission.4t.com/Physics/gtd.htm 
Scientific Research Publishing (SCIRP) is one of the largest Open Access journal publishers. It is currently publishing more than 200 open access, online, peer-reviewed journals covering a wide range of academic disciplines. SCIRP serves the worldwide academic communities and contributes to the progress and application of science with its publication.

Other selected journals from SCIRP are listed as below. Submit your manuscript to us via either submit@scirp.org or Online Submission Portal.
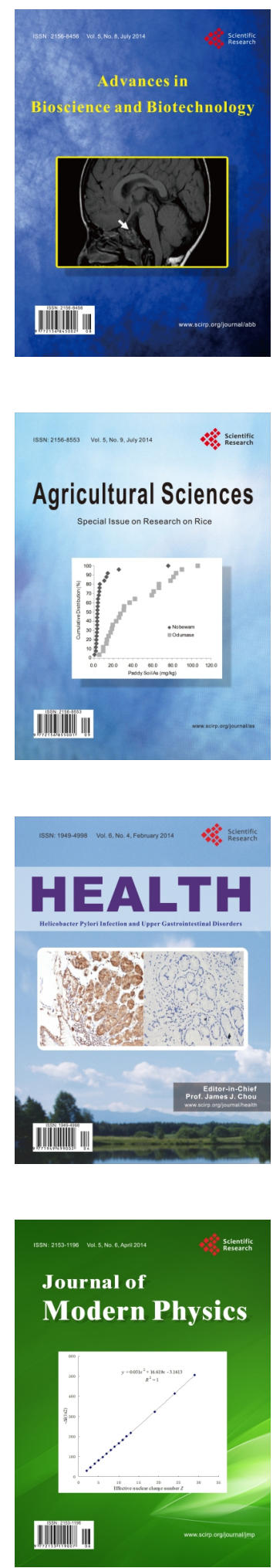
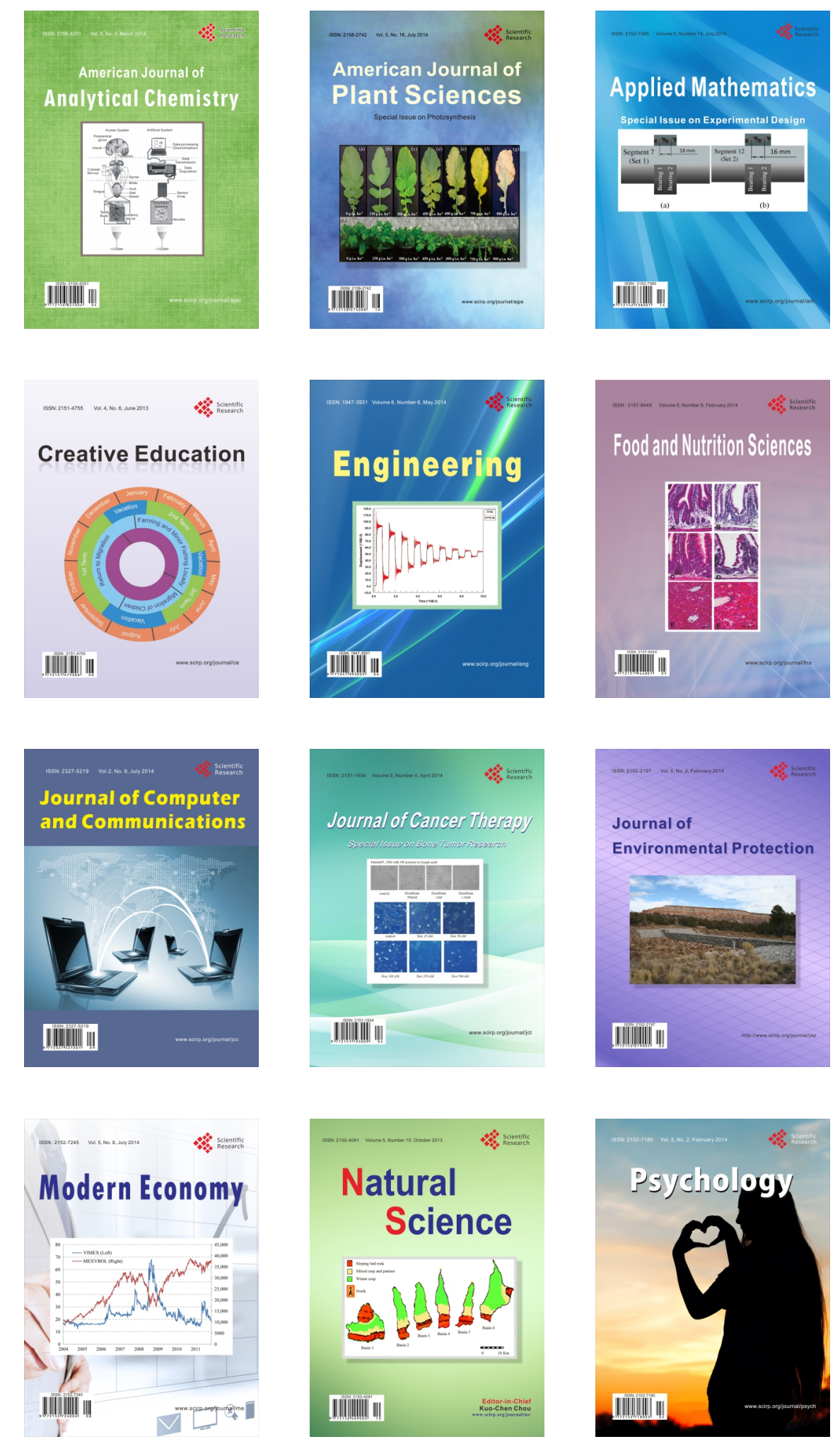